\title{
Undulatory and Pedundulatory Robotic Locomotion via Direct and Retrograde Body Waves
}

\author{
Michael Sfakiotakis and Dimitris P. Tsakiris
}

\begin{abstract}
The present paper explores the effect of the mechanism-substrate frictional interface on the locomotion characteristics of robotic mechanisms employing traveling waves for propulsion. For these investigations, an extended class of undulatory robotic locomotors is considered, termed pedundulatory, which augment lateral body undulations by coordinated dorso-ventral oscillations of multiple pairs of lateral paddle-shaped appendages (parapodia). We examine how, the same robotic prototype, allows the implementation of four distinct bio-inspired undulatory and pedundulatory modes of locomotion, by modifying the motion control strategy depending on the mechanism-substrate frictional interface. These modes employ retrograde or direct body waves, either standalone (giving rise to eel-like and ochromonas-like undulatory locomotion modes, respectively), or combined with appropriately coordinated substrate contact by the parapodial appendages (giving rise to centipede-like and polychaete-like pedundulatory modes, respectively). These four modes are investigated and comparatively assessed, both in simulation and via extensive experiments on granular substrates with the Nereisbot prototype. Our results validate the identified locomotion principles and also highlight the enhanced performance and gait repertoire of pedundulatory systems, compared to purely undulatory ones.

Keywords - biomimetic robotics, undulatory locomotion, motion control, eels, snakes, polychaete annelids, centipedes
\end{abstract}

\section{INTRODUCTION}

Undulatory locomotion presents considerable potential for increasing the mobility of robotic systems operating in challenging environments [1]-[8]. Extending our previous studies in this area [9]-[13], the present paper describes computational and experimental investigations of the effect of the mechanism-substrate frictional interface on the locomotion characteristics of robotic mechanisms which employ traveling body waves for propulsion. More specifically, it is demonstrated, both via simulations and via experiments over granular substrates with the Nereisbot robotic prototype [9], how an undulatory system may move forward using either retrograde (i.e., propagating from the head to the tail) or direct (i.e., propagating from the tail to the head) body waves, by reversing the frictional differential with respect to resistance in the tangential and normal directions encountered by the mechanism's segments. In addition, two pedundulatory modes are presented, in which these two types

This work was supported in part by the European Commission, through the EU R\&D projects VECTOR (FP6-033970) and OCTOPUS (FP7231608).

The authors are with the Institute of Computer Science, Foundation for Research and Technology - Hellas, N. Plastira 100, Vassilika Vouton, GR-70013, Heraklion, Greece. The first author is also with the Dept. of Electrical Engineering, Technological Educational Institute of Crete, Heraklion, Greece. \{sfakios, tsakiris\}@ics.forth.gr

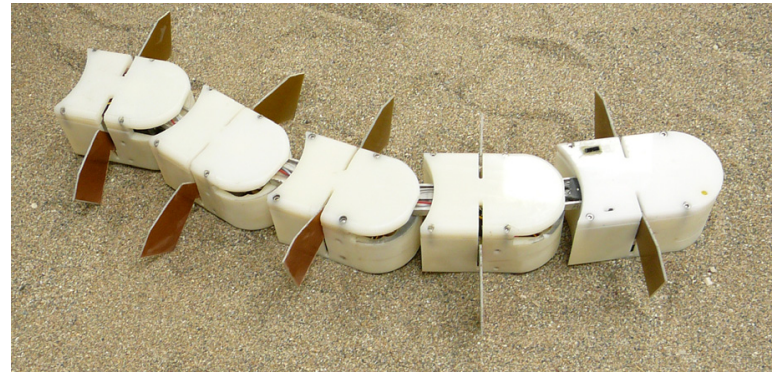

Fig. 1. The Nereisbot robotic prototype.

of body undulations are combined with appropriately coordinated motions of several lateral paddle-like appendages (termed parapodia), to significantly enhance the locomotive performance.

Our studies demonstrate how a single multi-segment robotic platform may generate a variety of undulatory-based locomotion modes, by altering the mechanism-substrate frictional interface and appropriately modifying the motion control strategy. Such an ability could have a significant impact on the terrain traversing capabilities of systems developed for a series of emerging robotic applications, ranging from novel medical devices for endoluminal diagnosis/intervention, to robotic systems for search-and-rescue operations and to planetary exploration.

It is worth noting that these undulatory and pedundulatory modes have been inspired by (rather than exactly replicating) the locomotion of biological organisms, spanning a broad range of body sizes (from sub-millimeter to meters), body plans (vertebrates, invertebrates, arthropods and protozoa) and habitats (terrestrial, marine and in viscous media).

Sections II and III of the paper describe models of the mechanics and motion control for the developed undulatory and pedundulatory locomotion modes. Section IV presents the Nereisbot pedundulatory robotic prototype (Fig. 1), which is employed to experimentally demonstrate the investigated motion control strategies, for locomotion over sand. The obtained results are presented in Section V and also verify that our computational models capture adequately essential features of the developed locomotion strategies.

\section{COMPUTATIONAL MODELING}

\section{A. Mechanism Model}

A mechanical model of the Nereisbot pedundulatory prototype, employed to study the proposed locomotion strategies, is shown in Fig. 2. It is composed of five links (link-1 is 


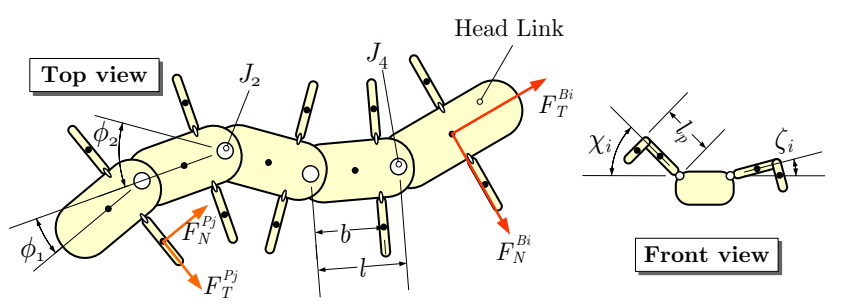

Fig. 2. Model of the Nereisbot robotic prototype.

the tail, and link-5 is the mechanism's head), interconnected by a total of four planar rotary joints $J_{i}$, whose activelycontrolled joint angles are denoted by $\phi_{i}$, while the distance between two consecutive joints is denoted by $l$. On each segment, a pair of laterally placed parapodial links of length $l_{p}$ is mounted at a distance $b$ from the segment's leftmost edge. The parapodia are connected to the segment via planar dorsoventrally-oriented single-degree-of-freedom rotary joints, whose angles to the horizontal are denoted by $\zeta_{i}$ and $\chi_{i}$, for the left-hand and right-hand parapodium of the $i$ th segment, respectively. By retracting all parapodia, the mechanism is essentially reduced to an undulatory system. In pedundulatory modes, the overall desired "paddling" action of the parapodia emerges when their up-down movements are combined with body undulations.

A computational model of the full body dynamics of the above system, incorporating the mechanical parameters (masses, dimensions, etc.) of the actual Nereisbot prototype, has been implemented in the SIMUUN simulation environment [12], which is based on the SimMechanics toolbox of Matlab/Simulink.

\section{B. Interaction with the Environment}

The frictional interaction of the undulatory mechanism's $i$ th body segment $\left(B_{i}\right)$ with the environment is described here by a Coulomb model, involving the decoupled components

$$
F_{T}^{B i}=-\mu_{T} m g \operatorname{sgn}\left(v_{T}^{B i}\right) \text { and } F_{N}^{B i}=-\mu_{N} m g \operatorname{sgn}\left(v_{N}^{B i}\right),
$$

acting in the tangential and normal direction of segment motion (see Fig. 2), where $v_{T}^{B i}$ and $v_{N}^{B i}$ are the respective velocity components, while $m$ is the segment's mass and $g$ is the constant of gravity. The effect of the tangential $\left(\mu_{T}\right)$ and normal $\left(\mu_{N}\right)$ Coulomb friction coefficients, which is of key significance for the development of the investigated locomotion modes, is considered in conjunction with motion control in Section III.

Regarding the parapodia of the system (which are only employed in pedundulatory modes), the simple Coulomblike frictional force model

$$
F_{T}^{P j}=-f_{T}^{i} \operatorname{sgn}\left(v_{T}^{P j}\right) \text { and } F_{N}^{P j}=-f_{N}^{i} \operatorname{sgn}\left(v_{N}^{P j}\right),
$$

may be used (as a first approximation) for describing the forces generated by the $j$ th parapodium $\left(P_{j}\right)$ when in contact with the specific locomotion environment (Fig. 2). The magnitudes $f_{N}^{i}$ and $f_{T}^{i}$ of the frictional forces, in the normal and tangential direction, respectively, can be determined experimentally for each pair of parapodia placed on the robot's $i$ th segment.

\section{MOTION CONTROL STRATEGIES}

This Section presents the motion control strategies, which have been developed for the different undulatory and pedundulatory modes under investigation.

\section{A. Undulatory Locomotion Modes}

In purely undulatory modes (i.e., with all parapodia retracted) locomotion is obtained through the coupling of the mechanism's internal shape changes (the traveling body wave) to external motion constraints (the frictional forces applied through the interaction with the environment). In the present study, the latter are described by the Coulomb friction model (1), where the tangential $\left(\mu_{T}\right)$ and normal $\left(\mu_{N}\right)$ friction coefficients depend on the shaping and material of the links' underside, as well as on the material properties of the substrate. In general, the larger the differential between these coefficients, the larger the stride length (distance traveled per undulation cycle) attainable for a given body wave. The friction coefficients' ratio also determines, to a large extent, the movement direction of the undulatory locomotor with respect to the direction of the body wave, as analyzed next (for details, see [11] and refs. therein).

Eel mode: When resistance to lateral segment motions is (considerably) higher than resistance to tangential motions, body undulations will generate an overall motion of the mechanism in the direction opposite to that of the body wave. Most undulatory robots (e.g., [1]-[7], [13]) implement this eel-like mode of undulatory locomotion by retrograde body waves, which is also by far the most common in nature, both on land (e.g., snakes) and in the water (e.g., eels).

Assuming full position control of our mechanism's joint angles, a straightforward method of obtaining such a headto-tail body wave, involves specifying a sinusoidal variation for the $i=1, \ldots, N-1$ body joint angles

$$
\phi_{i}(t)=A \sin \left(2 \pi f t+(N-i) \phi_{\text {lag }}\right)+\psi,
$$

with the same amplitude $A$, frequency $f$, angular offset $\psi$, and a constant phase lag $\phi_{\text {lag }}>0$ between consecutive joints. Therefore, for the present force model, when $\mu_{N} / \mu_{T} \gg 1$, motion control (3) gives rise to the eel undulatory mode.

Ochromonas mode: When the frictional interface is such that resistance to lateral segment motions is smaller than resistance to tangential ones, the overall locomotion is along the propagation direction of the undulatory wave. Relevant analysis and robotic implementations of systems obtaining forward motion by tail-to-head body waves are available in [8], [9], [11], [14]. Although rare, undulatory locomotion by such direct waves is also encountered in nature, e.g., in the propulsion of microorganisms with hispid flagella, such as the Ochromonas protozoan [15], [16]. The surface of hispid flagella bears rigid, laterally-protruding hair-like structures, by virtue of which, resistance in the tangential direction is greater than in the normal direction. 
The corresponding formulation for generating a tail-tohead body wave with the Nereisbot model is

$$
\phi_{i}(t)=A \sin \left(2 \pi f t+i \phi_{\text {lag }}\right)+\psi, i=1, . ., N-1 .
$$

Hence, for the present force model, when $\mu_{N} / \mu_{T}<1$ (or even when $\mu_{N} / \mu_{T} \simeq 1$, see [8], [11], [14]), motion control (4) will generate this ochromonas-like undulatory mode.

For both (3) and (4), when the angular offset is $\psi=0$, the mechanism moves along a straight (on the average) line, while curved paths are obtained for $\psi \neq 0$.

\section{B. Pedundulatory Locomotion Modes}

In pedundulatory modes, body undulations are combined with the periodic activation of the lateral parapodial appendages, which occurs in waves propagating in the same direction as the body undulations. Appropriate coordination is required, in order to ensure the positive contribution of the parapodia in thrust generation during each locomotion cycle. Two main such pedundulatory modes have been developed [9], [10], which are inspired by the locomotion of centipedes (for retrograde wave propagation) and of polychaete marine worms (for direct wave propagation). Note that, due to its mechanical design, our system (Fig. 2) cannot propel itself by the simple dorso-ventral parapodial movements alone; it is the traveling body wave that effectively positions the parapodia, allowing them to move backwards with respect to the substrate, and to thus impart propulsive forces.

Centipede mode: Centipedes combine, for forward locomotion, head-to-tail undulations of their segmented body with leg movements, synchronized so that the legs make contact with the substrate when the corresponding body segment is in the trough of the body wave [17]. The resultant periodic activity of the legs occurs in waves moving from the anterior to the posterior, along the two sides of the animal. A straightforward way to implement this centipede-like mode of pedundulatory locomotion with the mechanical system at hand (Fig. 2), involves combining the head-to-tail body wave (3) with the alternating activation of the right and left parapodium of the $j$ th body segment as follows (note that the parapodia are in contact with the substrate for $\chi_{j}(t)=0$ and $\zeta_{j}(t)=0$, respectively):

$\chi_{j}(t)=\left\{\begin{array}{ll}0, & \text { for } \beta_{j}(t)<-r_{j} \\ A_{p}, & \text { otherwise }\end{array}, \zeta_{j}(t)=\left\{\begin{array}{ll}0, & \text { for } \beta_{j}(t)>r_{j} \\ A_{p}, & \text { otherwise }\end{array}\right.\right.$,

$$
\text { for } \beta_{j}(t)=\sin \left(2 \pi f t+\left(j-g_{j}^{c}\right) \phi_{\text {lag }}\right), j=1, . ., N \text {, }
$$

where $r_{j}$ is an appropriate threshold $\left(0<r_{j}<1\right)$ and the retraction angle $A_{p}$ is specified to allow the parapodia to be fully lifted off the substrate. The periodic movements of each segment's parapodia are synchronized with the body wave via the sinusoidal reference signal $\beta_{j}(t)$. The latter is derived by the (scaled) corresponding body joint angle $\phi_{i}(t)$, appropriately shifted in time via the $g_{j}^{c}$ parameter, to ensure its temporal variation corresponds to that of the parapodium velocity, resolved along the mean direction of propulsion. The parameter $g_{j}^{c}$ primarily depends on the relative placement of the parapodia on the segment with respect to the joint (parameter $b$ in Fig. 2).

Polychaete mode: Each body segment of the polychaete marine worms features a pair of laterally-extending, paddleshaped parapodia. When crawling rapidly or swimming, polychaete combine tail-to-head body undulations with alternating waves of parapodial activity, coordinated so that the thrust-producing power stroke of each parapodium occurs at the crest of the body wave, while its recovery phase occurs in the trough of the body wave [18], [19]. To implement this polychaete-like pedundulatory mode, the tail-to-head body wave (4) is combined with the following scheme of parapodia activation:

$\chi_{j}(t)=\left\{\begin{array}{cl}0, & \text { for } \beta_{j}(t)>r_{j} \\ A_{p}, & \text { otherwise }\end{array}, \quad \zeta_{j}(t)= \begin{cases}0, & \text { for } \beta_{j}(t)<-r_{j} \\ A_{p}, & \text { otherwise }\end{cases}\right.$

for $\beta_{j}(t)=\sin \left(2 \pi f t+\left(N+g_{j}^{p}-j\right) \phi_{l a g}\right), j=1, . ., N$.

For both pedundulatory modes, turning motions may be instigated by a number of different ways, e.g., (a) by introducing an angular offset $\psi \neq 0$ in the body wave (3) or (4), while the parapodia operate in the normal bilateral fashion of the corresponding pedundulatory mode, $(b)$ by unilateral parapodial activations (i.e., by retracting all parapodia in the side of the mechanism appropriate for the desired turning direction), while $\psi=0$, and (c) by combining an angular offset $\psi \neq 0$ with unilateral parapodial activation.

More details on the mechanism's kinematics for the proposed pedundulatory modes are available in [10].

\section{Simulations}

Simulations were carried out for the four investigated modes of undulatory and pedundulatory locomotion, involving the SIMUUN Nereisbot model. Data from appropriate frictional force measurements (see Section V) were employed to specify the parameters of the force models (as per Section II-B) for the interaction with the environment of the body segments (i.e., coefficients $\mu_{N}$ and $\mu_{T}$ ) and of the parapodia (i.e., coefficients $f_{N}$ and $f_{T}$ ). These are shown in Fig. 3, along with indicative trajectories for the forward gait. Furthermore, the results of a parametric study with this simulation setup are provided in Figs. 7c and 9c, in the context of comparing them with the experimental data.

\section{THE NEREISBOT ROBOTIC PLATFORM}

The proposed undulatory and pedundulatory locomotion strategies are investigated experimentally with the Nereisbot robotic prototype [9], [10]. This comprises five body segments, each equipped with a pair of parapodial appendages, providing a total of 14 independently-controlled degrees-offreedom (corresponding to 4 body joints and 10 parapodial joints). The first four body segments are identical (length $100 \mathrm{~mm}$, width $50 \mathrm{~mm}$, mass $270 \mathrm{~g}$ ), and each one encases three $\mathrm{R} / \mathrm{C}$ servomotors (Fig. 4a), actuating the main body joint (through which consecutive segments are connected together) and the segment's pair of parapodia. The fifth, "head" segment of the robot does not require a body joint 


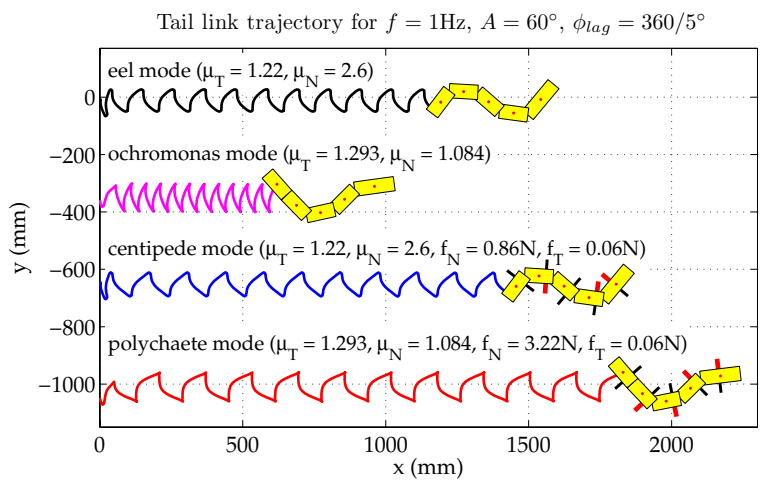

Fig. 3. Forward gait simulations of Nereisbot implementing the two undulatory and the two pedundulatoy modes $(12$ cycles shown for each mode, tracing the trajectory of the tail joint $J_{1}$ ). The respective force coefficients, used in the simulations, are also indicated for each mode. For the two pedundulatory modes, the legs in contact with the substrate (at the selected snapshots of the mechanism's motion) are denoted in red.

servo, and has been made longer (length $140 \mathrm{~mm}$ ), in order to accommodate the robot's control unit.

The parapodia (Fig. 4) are directly mounted on the shaft of their actuating motor so that their flat surface is lateral to the segment's main axis. Their shape allows efficient penetration of granular substrates, with a nominal effective area (estimated at $\sim 1280 \mathrm{~mm}^{2}$ ) about 2.4 times that of the parapodia employed in [9]. A special instrumented parapodium unit has also been developed [9], [10], which incorporates strain gauges for measuring the normal component $F_{N}^{P j}$ of the force generated by the paddling parapodial motions (see Fig. 6b).

When crawling over granular substrates on the body segments' flat underside, forward undulatory propulsion of Nereisbot is obtained via tail-to-head body waves [9], [10]. The characteristics of the robot's frictional interaction with the locomotion environment (and hence the propulsive mode and/or performance) may be altered by attaching different modules on the underside of the body segments. For example, forward undulatory propulsion by retrograde waves may be obtained, for conventional surfaces (e.g., hard floor), via longitudinally-mounted wheeled modules [13].

For the present study of locomotion over granular substrates, two different body morphologies are considered for Nereisbot, characterized by a reversed friction differential for the robot's underside. The first body morphology (henceforth termed BM-L) utilizes long aluminium blades (length: $90 \mathrm{~mm}$ ), mounted longitudinally across the main

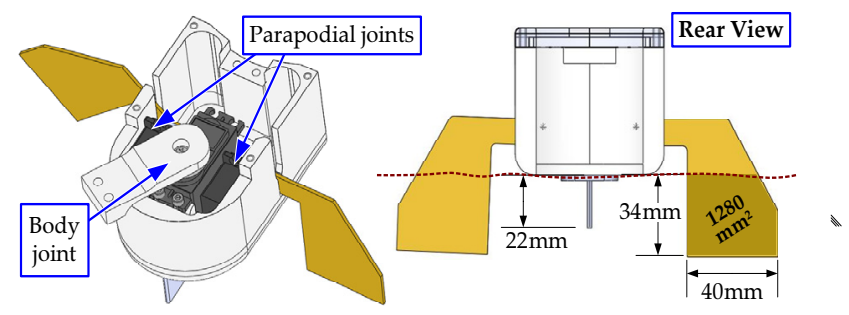

(a)

(b)

Fig. 4. CAD diagrams of a Nereisbot body segment, illustrating (a) the arrangement of the three servo motors and (b) the penetration of granular substrates by the parapodia and the blade attachment (here shown to be longitudinally mounted on the underside of the segment).

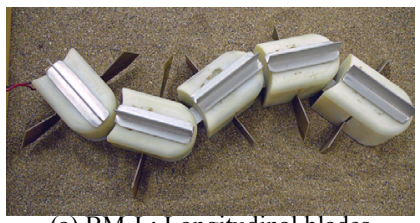

(a) BM-L: Longitudinal blades

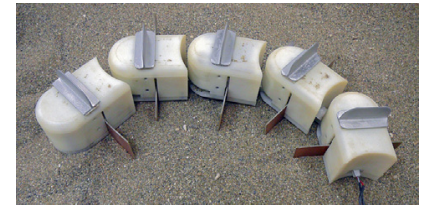

(b) BM-T: Transversal blades
Fig. 5. Configuration of the robot's underside for the two body morphologies employed in the experiments.

axis of the robot's five segments (see Fig. 5a), in order to provide increased frictional resistance to normal segment motions. The second body morphology (henceforth termed BM-T) employs shorter blades (length: $57 \mathrm{~mm}$ ), mounted transversely with respect to the segments' main axis (Fig. $5 b)$, increasing the frictional resistance to tangential segment motions. The height of both blade variants is $22 \mathrm{~mm}$.

\section{EXPERIMENTS ON SAND}

Experimental results from tests with the Nereisbot prototype on sand are presented here, demonstrating the generation of the four locomotion modes identified in Section III, utilizing the two body morphology configurations of Fig. 5. Comparisons with the results of corresponding SIMUUN simulations are also provided.

Experiments were conducted inside a box (measuring $1.7 \mathrm{~m} \times 1.7 \mathrm{~m}$ ) uniformly filled with about $55 \mathrm{~mm}$ of fine seashore sand (mean particle diameter: $0.6 \mathrm{~mm}$ ). The robot was powered by an external $7.0 \mathrm{~V}$ power supply, while colored markers enabled the reconstruction of the joints' trajectories, via post-processing of the experiment videos (samples of which are provided in the accompanying video).

Finally, for assessing the developed computational models, corresponding simulations were carried out in SIMUUN, replicating, for each experimental run, the set of parameters used, and incorporating the frictional data below.

\section{A. Frictional Interface Characterization}

In order to select an appropriate force model for the simulations, special measurements were performed to obtain data on the frictional interface between the mechanism and the locomotion substrate (i.e., sand), for both of the configurations of the segments' underside (corresponding to BM-L and BM-T). A custom test-stand, shown in Fig. 6a, was used to measure the forces required to move a single body segment over sand, in both the tangential and normal directions with respect to its main axis. The test stand is based on a motorized linear stage, on which a high-precision digital force gauge (Alluris FMI-210A5) is mounted. The gauge provides data regarding the frictional resistance of the segment, as the latter is pulled over the environment of choice at different traction velocities.

Much like the corresponding tests in [9], [11], the measured forces presented little variance in the range of traction velocities tested, indicating that a simple Coulomb friction model could be used for the interaction of the body with the sand environment. The resultant sets of Coulomb friction coefficients (summarized in Table I) verify that the use of 
TABLE I

FRICTION FORCE COEFFICIENTS FOR THE FLAT-UNDERSIDE AND FOR THE TWO BLADE-BEARING CONFIGURATIONS OF THE ROBOT.

\begin{tabular}{c|c|c|c}
\hline \hline & $\mu_{T}$ & $\mu_{N}$ & $\mu_{T} / \mu_{N}$ \\
\hline \hline Without blades (flat underside) & 0.518 & 0.463 & 1.111 \\
BM-L: long blades, longitudinally mounted & 1.22 & 2.6 & $(2.16)^{-1}$ \\
BM-T: short blades, transversally mounted & 1.293 & 1.084 & 1.193 \\
\hline$\mu_{T}:$ tangential friction coefficient, $\mu_{N}:$ normal friction coefficient \\
\hline \hline
\end{tabular}

blades significantly increases the magnitude of the forces resisting motion, compared to the flat-underside configuration. The blade elements employed in the present study provide a (considerably) increased friction differential, compared to the blade designs used in our prior work [9], [11], due to their increased height, which allows a higher degree of substrate penetration. This was found to be an important feature for generating the eel mode of undulatory locomotion (see Section V-B). As expected, the longer blades are associated with higher frictional forces when moved laterally against the substrate (corresponding to $\mu_{N}$ for BM-L in Table I), compared to the shorter blades (corresponding to $\mu_{T}$ for the BM-T configuration in Table I).

For describing the interaction of the parapodia with sand, the simple Coulomb-like frictional model (2) is used. A series of measurements, obtained with the instrumented parapodium mounted on the tail segment of the mechanism (Fig. 6b), yielded an average value for the magnitude $f_{N}^{i}$ $(i=1)$ of the frictional force in the normal direction equal to $3.22 \mathrm{~N}$ for the polychaete mode, and equal to $0.86 \mathrm{~N}$ for the centipede mode (see Section V-C for an explanation for this pronounced difference between the two modes). These values were used to specify $f_{N}^{i}$ in (2) for all of the mechanism's parapodia, in simulations of the corresponding pedundulatory modes. Due to the shape and movement of the parapodia, the magnitude $f_{T}$ of the tangentially-oriented frictional forces may be considered negligible.

\section{B. Undulatory Modes}

Tests involving pure undulations of the Nereisbot prototype (i.e., with all parapodial activity disabled), were conducted over a range of values for $A\left(20^{\circ}-60^{\circ}\right)$, with $f=1 \mathrm{~Hz}$. The obtained results verified the main qualitative prediction of our computational models, related to the effect

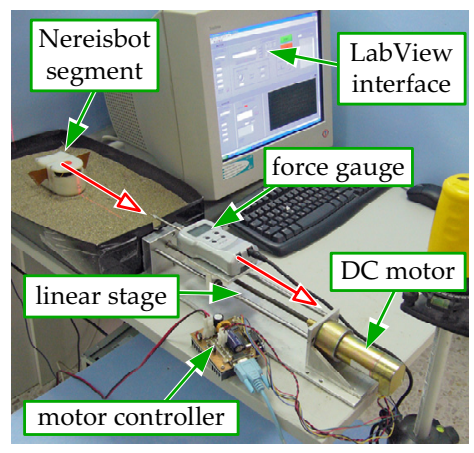

(a)

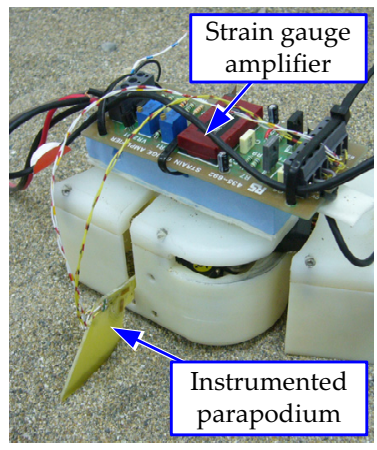

(b)
Fig. 6. (a) The test-stand utilized for the body segment frictional force measurements. (b) Setup for the parapodia force measurements.

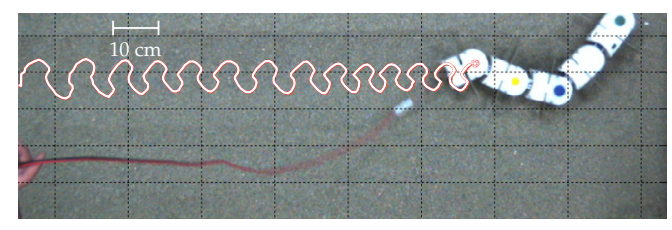

(a) BM-L: eel mode of undulatory locomotion $\left(A=60^{\circ}\right)$

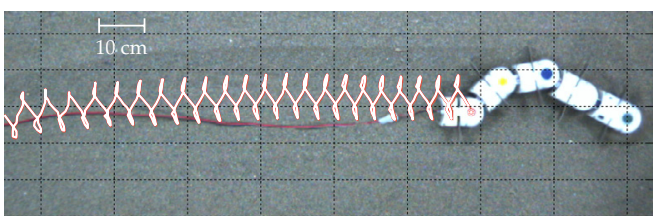

(b) BM-T: ochromonas mode of undulatory locomotion $\left(A=60^{\circ}\right)$

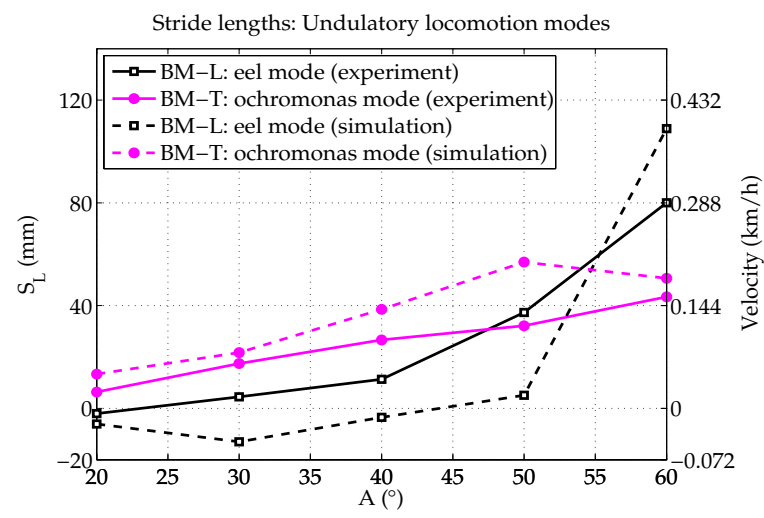

(c) summary of stride length results

Fig. 7. Forward undulatory modes, obtained with the two body morphologies: (a)-(b) Nereisbot experimental results, indicating the trajectory of the tail joint $J_{1}$. (c) Experimental (solid lines) vs. simulation-derived (dashed lines) stride length and velocity estimates of the robot (negative $S_{L}$ values indicate backwards movement).

of reversing the frictional differential between the normal and the tangential direction of motion. More specifically, these experiments demonstrated that, for the BM-L configuration of the robot, forward propulsion is obtained by retrograde body waves (i.e., when (3) is employed for motion control), while for the BM-T configuration, forward motion of the robot is obtained by the propagation of direct body waves (i.e., when (4) is employed for motion control). Indicative robot trajectories are provided in Fig. 7(a)-(b), while the stride lengths $S_{L}$ (and equivalent average velocities $v=$ $f S_{L}$ ) attained by the robot, along with the corresponding results of the SIMUUN simulations, are summarized in Fig. 7 (c). The attained stride lengths were found to increase with the amplitude of joint oscillations (for the range of $A$ values tested), obtaining their maximum values of $80 \mathrm{~mm}$ (for the eel mode) and $44 \mathrm{~mm}$ (for the ochromonas mode) for $A=60^{\circ}$. On a comparative basis, it may be observed that the performance of the ochromonas mode is generally more consistent throughout the range of $A$ values tested, and attains higher stride lengths than the eel mode for values of $A$ up to $40^{\circ}$; however, as $A$ is further increased, there is a steep increase in the performance of the eel mode. It is noteworthy that these observations may also be (qualitatively) identified in the simulation results.

Indicative results from turning gait experiments are pro- 


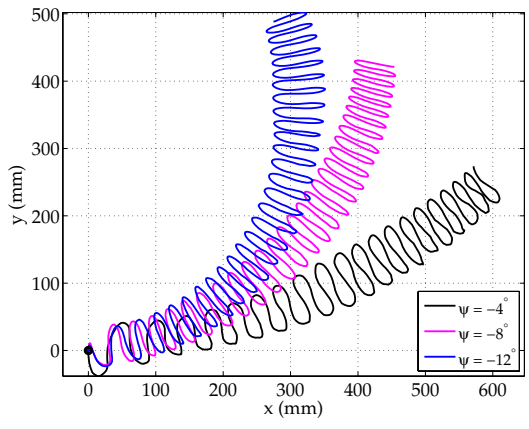

(a) BM-L: eel mode

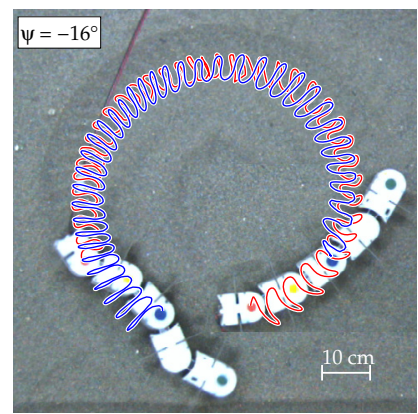

(c) BM-L: eel mode $\left(A=50^{\circ}\right)$

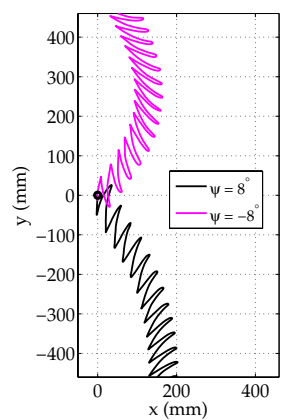

(b) BM-T: ochromonas mode

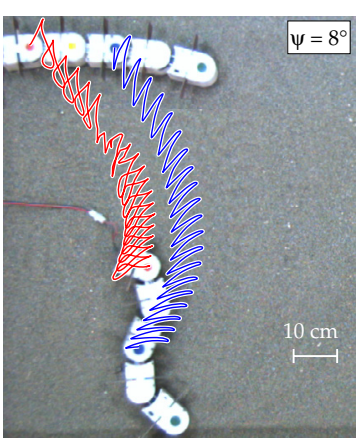

(d) BM-T: ochromonas mode $\left(A=50^{\circ}\right)$
Fig. 8. Turning undulatory gait for the two body morphologies: Nereisbot experimental results (a)-(b) Effect of the joint offset angle $\psi$ on the turning directions and turning radius for the two body morphology configurations. The plots indicate the trajectories traced by the middle joint $J_{3}$ of the robot. (c)-(d) Plots highlighting the differences, with regard to the turning gait, for the two undulatory modes. The trajectories of the tail $\left(J_{1}\right.$, in red) and middle $\left(J_{3}\right.$, in blue) joints of the robot are shown.

vided in Fig. 8, where the effect of the joint angle offset $\psi$ on the turning direction and turning radius of the robot is demonstrated. It may also be observed that, for retrograde wave propulsion (BM-L configuration), all segments of the mechanism follow a common mean path (Fig. 8c); by contrast, for direct wave propulsion (BM-T configuration), there is a distinct offset in the mean trajectories of the mechanism's segments during turning motions (Fig. 8d). These characteristic features of the two undulatory modes were also observed in previous experimental investigations [11], and are consistent with simulation results.

\section{Pedundulatory Modes}

Experiments, involving the combined use of parapodial activity and body undulations, verified the proposed pedundulatory locomotion strategies, employing the BM-L configuration of the robot to implement the centipede mode via (3) and (5), and the BM-T configuration to implement the polychaete mode, via (4) and (6). For both modes, guidelines for specifying the values of $g_{j}^{c}, g_{j}^{p}$ and $r_{j}$ in (5) and (6), were provided by simulations [10]. Indicative forward gait trajectories, obtained with the two pedundulatory modes, are shown in Fig. 9(a)-(b), while estimates of the robot's attained stride lengths, both experimental and simulationderived, are summarized in Fig. 9(c). Much like for the undulatory modes, higher $S_{L}$ values were obtained as the amplitude $A$ of the body joint oscillations was increased,

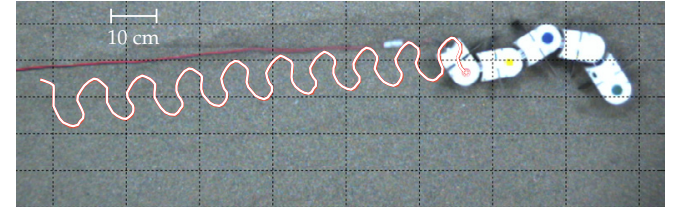

(a) BM-L: centipede mode of pedundulatory locomotion $\left(A=60^{\circ}\right)$

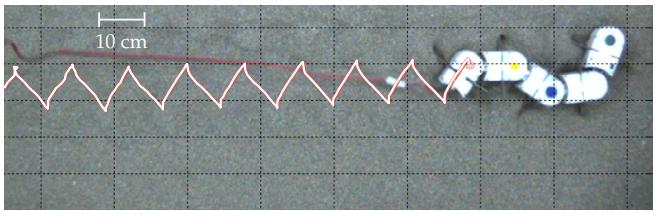

(b) BM-T: polychaete mode of pedundulatory locomotion $\left(A=60^{\circ}\right)$

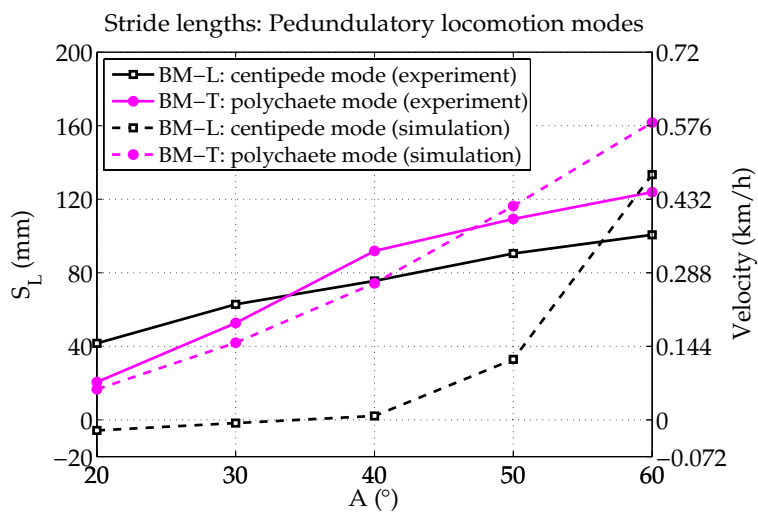

(c) summary of stride length results

Fig. 9. Forward pedundulatory modes, obtained with the two body morphologies: (a)-(b) Nereisbot experimental results, indicating the trajectory of the tail joint $J_{1}$. (c) Experimental (solid lines) vs. simulation-derived (dashed lines) stride length and velocity estimates of the robot.

obtaining their maximum values (124 $\mathrm{mm}$ for the polychaete, and $101 \mathrm{~mm}$ for the centipede mode) when $A=60^{\circ}$. One possible cause for the comparatively reduced performance of the centipede mode, also related to the difference between the measured parapodial forces (Section V-A), was the fact that the longitudinally-mounted blades in the BM-L configuration were observed to shift a significant amount of sand away from the parapodia, essentially reducing the effective (i.e., thrust-producing) area of the parapodia.

The different strategies for pedundulatory turning motions, identified in Section III-B, have also been experimentally validated. Indicative trajectories are shown in Fig. 10.

\section{Discussion}

The obtained results have validated the underlying locomotion principles of the proposed bio-inspired undulatory and pedundulatory modes, providing illustrative demonstrations of how the mechanism-substrate frictional interface imposes adjustments to the motion control strategy. In particular, the present study is, to the best of our knowledge, the first one to demonstrate undulatory locomotion by either direct or retrograde body waves propagating along a common mechanical platform, obtained via reversal of the frictional differential in the tangential and normal directions of segment motion. In contrast to our previous research efforts [9], [11], this has been made possible via the increased friction 


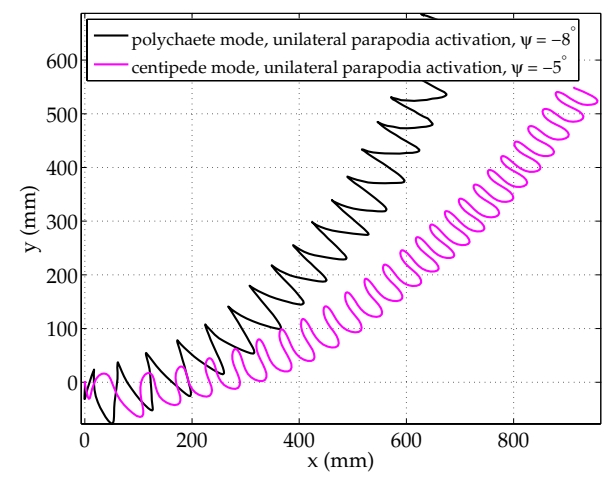

Fig. 10. Turning polychaete-like pedundulatory gait: Nereisbot experimental results for $A=50^{\circ}$, showing the trajectory of the central joint $J_{3}$.

differential afforded by the re-designed blade elements.

The experiments also highlight the enhanced performance and gait repertoire of the pedundulatory modes, compared to the respective undulatory ones (i.e., when considering centipede $v s$. eel and polychaete $v s$. ochromonas modes). In particular, there is a nearly three-fold increase in the maximum attained velocities between the polychaete $(0.45$ $\mathrm{km} / \mathrm{h})$ and ochromonas modes $(0.16 \mathrm{~km} / \mathrm{h})$.

Our computational models have been, on the whole, successful in capturing the main qualitative characteristics of the investigated locomotion strategies, and provide adequate quantitative predictions for the robot performance. Any inconsistencies with the experimental data could be attributed, at least in part, to the simplified nature of the employed friction models, which cannot describe the whole range of complex phenomena and interactions, tribological or otherwise (e.g., sand shifting or sand compaction), between the robot and the highly unstructured nature of sand. Such interactions may be further intensified by the large height of the employed blades (as opposed to experiments performed using the flat-underside configuration of the robot [10]), further degrading the applicability of our computational models. An additional source of discrepancies is the use of the same $f_{N}^{i}$ value in (2) for all of the mechanism's parapodia, based on data obtained from a single location (the tail segment). Extensive tests are currently underway with the instrumented parapodium, in order to acquire more comprehensive data regarding the force profile of individual parapodia; these are expected to provide additional insights regarding the force generation of parapodia as a function of their position across the mechanism's body, and to improve the accuracy of our simulation tools.

\section{CONCLUSIONS}

The effect of the frictional interface of multi-segment undulatory locomotors with their substrate was studied. This interface is implemented, for granular substrates, by blade modules, located on the underside of each segment, and oriented longitudinally or transversally with respect to the segment axis, and by parapodial appendages protruding laterally from each segment. Several bio-inspired modes of locomotion, due to the interaction of a direct or retrograde body wave with the orientation of the blades, and, in the presence of parapodia, with the synchronization of their oscillations to the body wave, were demonstrated, both in simulation and experimentally, to be generated from the same mechanism. The enhanced performance of the pedundulatory modes (with respect to the purely undulatory ones) was established for locomotion on fine sand, as well as the agreement of the experimental results with the predictions of our computational models. The development of additional, not necessarily bio-inspired, gaits (e.g., turning-in-place), with optimizations specific to each of these modes, is expected to further increase the locomotion abilities of our systems.

\section{ACKNOWLEDGMENTS}

The authors thank N. Pateromichelakis for his technical assistance. Related papers and videos can be found at the Web site www.ics.forth.gr/ tsakiris

\section{REFERENCES}

[1] S. Hirose, Biologically Inspired Robots: Snake-Like Locomotors and Manipulators. New York: Oxford University Press, 1993.

[2] J. P. Ostrowski and J. Burdick, "The geometric mechanics of undulatory robotic locomotion," Int. J. Robot. Res., vol. 17, no. 7, pp. 683-701, 1998

[3] P. S. Krishnaprasad and D. P. Tsakiris, "Oscillations, SE(2)-snakes and motion control: A study of the roller racer," Dyn. Syst., vol. 16, no. 4, pp. 347-397, 2001.

[4] K. Lipkin, I. Brown, A. Peck, H. Choset, J. Rembisz, P. Gianfortoni, and A. Naaktgeboren, "Differentiable and piecewise differentiable gaits for snake robots," in Proc. IEEE/RSJ Int. Conf. Intell. Robots Syst., San Diego, USA, 2007, pp. 1864-1869.

[5] M. Saito, M. Fukaya, and T. Iwasaki, "Modeling, analysis, and synthesis of serpentine locomotion with a multilink robotic snake," IEEE Control Syst. Mag., vol. 22, no. 1, pp. 64-81, 2002.

[6] J. Cortes, S. Martinez, J. P. Ostrowski, and K. A. McIsaac, "Optimal gaits for dynamic robotic locomotion," Int. J. Robot. Res., vol. 20, no. 9, pp. 707-728, 2001.

[7] S. Ma, "Development of a creeping locomotion snake-robot," Int. J. Robot. Automat., vol. 17, no. 4, pp. 146-153, 2002.

[8] A. A. Transeth, R. I. Leine, C. Glocker, and K. Y. Pettersen, "3-d snake robot motion: Nonsmooth modeling, simulations, and experiments," IEEE Trans. Robot., vol. 24, no. 2, pp. 361-376, 2008.

[9] M. Sfakiotakis, D. P. Tsakiris, and K. Karakasiliotis, "Polychaete-like pedundulatory robotic locomotion," in Proc. IEEE Int. Conf. Robot. Autom., Roma, Italy, 2007, pp. 269-274.

[10] M. Sfakiotakis and D. P. Tsakiris, "Pedundulatory robotic locomotion: Centipede and polychaete modes in unstructured substrates," in Proc. IEEE Int. Conf. Robot. Biomim. (ROBIO'08), (to appear).

[11] G. La Spina, M. Sfakiotakis, D. P. Tsakiris, A. Menciassi, and P. Dario, "Polychaete-like undulatory robotic locomotion in unstructured substrates," IEEE Trans. Robot., vol. 6, pp. 1200-1212, 2007.

[12] M. Sfakiotakis and D. P. Tsakiris, "SIMUUN: A simulation environment for undulatory locomotion," Int. J. Model. Simul., vol. 26, no. 4, pp. 4430-4464, 2006.

[13] , "A biomimetic centering behavior for undulatory robots," Int. $J$ Robot. Res., vol. 26, no. 11-12, pp. 1267-1282, 2007.

[14] M. Nilsson, "Serpentine locomotion on surfaces with uniform friction," in Proc. IEEE/RSJ Int. Conf. Intell. Robots Syst., Sendai, Japan, 2004, pp. 1751-1755.

[15] C. Brennen, "Locomotion of flagellates with mastigonemes," $J$. Mechanochem. Cell. Motility, vol. 3, pp. 202-217, 1975.

[16] M. E. J. Holwill and M. A. Sleigh, "Propulsion by hispid flagella," $J$. Exp. Biol., vol. 47, no. 2, pp. 267-276, 1967.

[17] B. Anderson, J. Shultz, and B. Jayne, "Axial kinematics and muscle activity during terrestrial locomotion of the centipede, Scolopendra heros," J. Exp. Biol., vol. 198, no. 5, pp. 1185-1195, 1995.

[18] J. Gray, "Annelids," in Animal Locomotion. London: Weidenfeld \& Nicolson, 1968, pp. 377-410.

[19] R. B. Clark and D. J. Tritton, "Swimming mechanisms in nereidiform polychaetes," J. Zool., vol. 161, pp. 257-271, 1970. 